Neurosurg Focus 25 (5):E16, 2008

\title{
Traumatic spine injuries in the geriatric population
}

\author{
Pascal Jabbour, M.D., ${ }^{1}$ Michael Fehlings, M.D., Ph.D., ${ }^{2}$ Alexander R. Vaccaro, M.D., \\ Ph.D., ${ }^{1}$ and James S. Harrop, M.D. ${ }^{1}$ \\ ${ }^{1}$ Departments of Neurological and Orthopaedic Surgery, Thomas Jefferson University, Philadelphia, \\ Pennsylvania; and ${ }^{2}$ Division of Neurosurgery and Spine Program, University of Toronto, Ontario, Canada
}

\begin{abstract}
In this paper the authors review spine trauma and spinal cord injury (SCI) in the geriatric population. The information in this study was compiled through a literature review of clinical presentation and management of SCI in the elderly population. This was done to define, identify, and specify treatment algorithms and management strategies in this unique patient population. (DOI: 10.3171/FOC.2008.25.11.E16)
\end{abstract}

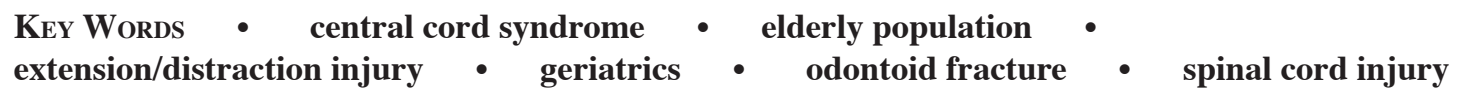

$\mathrm{T}$ RAUMATIC injuries to the spinal column and/or spinal cord can result in significant morbidity, disability, and even death. ${ }^{12} \mathrm{~A}$ common misconception is that these injuries occur in isolation and only to adolescents and young adults. However, epidemiological studies on populations with SCIs report a bimodal distribution. ${ }^{19}$ The first peak occurs as expected in young adults and adolescents; however, the second peak occurs to the elderly population.

Geriatric patients are not clearly defined in the spine literature. For this manuscript, the term will be defined as individuals $>65$ years of age. In North America, this population is particularly important in health care because it is expected to double over the next 30 years, from 34 million in 1999 to 69 million in $2030 . .^{22}$ In fact, this "baby boomer population" had the largest percentage increase of any age group from 1990 to 2000. In this age group the leading cause of unexpected death is traumatic injuries and their associated complications, often as a result of injury to the spinal cord. ${ }^{25}$

The number of fall-induced injuries has increased as the general population has aged over the last decade. Sensory mechanisms instrumental in ambulation such as vision, vestibular system function, lower-extremity proprioception, and an upright posture are greatly diminished as patients age. ${ }^{24}$ Furthermore, chronic condi-

Abbreviations used in this paper: $\mathrm{CCS}=$ central cord syndrome; $\mathrm{ROM}=$ range of motion; $\mathrm{SCI}=$ spinal cord injury. tions encountered in the elderly present additional risk factors, such as osteoarthritis of the joints that results in decreased mobility, an inability to maneuver, and lessened postural stability. Neuromuscular and neurological disorders, such as Parkinson disease and diabetes, are associated with rigidity, slowed movement initiation, cognitive impairment, and decreased proprioception. In addition, elderly patients are typically on numerous medications that can impair sensation, coordination, and result in postural hypotension, all of which places patients at risk for falls and subsequent spinal injury. Therefore, the management of SCI in the geriatric population has to be undertaken with an understanding of these patients' unique pathophysiological characteristics and concerns for their age-related comorbidities.

Geriatric patients are vulnerable to traumatic SCI as a result of a number of factors, including the following: 1) osteoporosis, osteopenia, and natural changes in bone quality that occur with aging; 2) increasing prevalence of developmental cervical spinal stenosis due to spondylosis; 3) increased propensity for traumatic, related injuries (that is, falls) due to loss of sensory mechanisms and medication effects; and 4) a higher rate of motor vehicle collisions per mile driven.

Treatment of elderly patients with vertebral injuries and SCIs must be undertaken with an understanding of the unique pathophysiological characteristics, age-related disorders, and subsequent comorbidities that affect this patient population. Due to their limited physiological reserves and the existence of medical comorbidities, it is 


\section{P. Jabbour et al.}

believed that geriatric patients have an increased risk of morbidity and death from SCIs.

\section{Incidence and Mechanism of Injury}

In 1981, Bracken et al. ${ }^{3}$ noted that traumatic spinal cord lesions are more common in younger patient populations, and these investigators reported that only $20 \%$ of all SCIs occurred in persons $\geq 65$ years of age. In $<$ 25 years since that original publication, the frequency of traumatic SCIs appeared to have progressed significantly, particularly among elderly people. ${ }^{14}$ This increase appears to be the result of a significant growth in the total number of the aging population, in addition to the propensity of older patients to sustain SCIs with relatively minor trauma.$^{10}$ Kannus et al. ${ }^{16}$ reported that the total number of low-impact, fall-induced fractures with associated SCIs increased considerably from 1970 to 1995 . In their study they reported an increase from 60 to 419 patients and a mean increase of $24 \%$ annually. In particular, this demographic shift was particularly skewed toward elderly men. ${ }^{23}$ The high incidence of related injuries in men may be due to the relationship of cervical spondylosis and SCI, and to men's increased propensity compared with women (2:1 male predominance) to develop this disorder. ${ }^{11,13}$

Unlike SCIs in adolescents, which are related to high-velocity mechanisms, in patients $>45$ years of age the most common mechanism is a fall, which has been reported to account for as much as $77 \%$ of the cases. ${ }^{14}$ In the geriatric population, falls from heights $<1 \mathrm{~m}$ can result in severe SCIs. The elderly population has a predisposition to baseline spinal canal stenosis, and the resultant spinal cord compression from underlying degenerative cervical spondylosis, typically with concurrent osteopenia or osteoporosis. Motor vehicle collisions, although less common in the elderly population, remain the second most common mechanism of injury. Although the influence of alcohol or drugs is perceived to be limited to younger patients, Weingarden and Graham ${ }^{30}$ illustrated that $53 \%$ of patients with SCI who were $>50$ years of age were under the influence of alcohol prior to falling.

\section{Pathophysiological Features of Geriatric SCI}

Unfortunately, as a consequence of aging and spondylotic changes in the spinal column, geriatric patients are predisposed to traumatic SCI from even minor falls. Disc space collapse, osteophyte development, and ligamentum flavum buckling create degenerative spondylosis of the cervical spine. The consequences of these degenerative changes can result in stenosis of the canal, with resultant spinal cord compression. Teresi et al. ${ }^{31}$ reported a $26 \%$ incidence of cervical stenosis in patients $>65$ years of age based on MR imaging studies of asymptomatic individuals. In patients with advanced stenosis in whom there is loss of the cerebrospinal fluid cushion around the spinal cord, minor trauma can produce SCI without fractures or ligamentous injuries. Based on radiological criteria, $90 \%$ of men $>50$ years and $90 \%$ of women $>60$ years of age have evidence of degenerative changes in the cervical spine. $^{32}$
In addition, as the spinal column becomes more spondylotic it develops increased rigidity due to loss of ROM, particularly between the C4-5 and C5-6 levels. ${ }^{9}$ These degenerative changes result in the creation of a rigid lever arm, so that application of an external force on the spinal column (that is, trauma) concentrates the forces on a finite region, resulting in a fracture and SCI. The spine, being rigid, does not have the flexibility to accommodate such external forces, often resulting in a fracture or fracture and dislocation. These fracture risks are further increased due to the diminished vertebral bone quality secondary to osteoporosis from advanced age.

Advanced spondylotic degenerative disease of the cervical spine can create spinal canal stenosis, with resultant spinal cord compression. This spinal cord compression may be manifested clinically as a myelopathy, with impairment of the patient's gait, coordination, bowel or bladder function, and motor and/or sensory function. These neurological deficits predispose the affected geriatric patients to an even higher risk for falls and SCIs.

Schneider et al. ${ }^{27}$ first described the clinical spinal cord incomplete syndrome, which they referred to as a CCS, as motor loss where the hands are more affected than the legs. These authors noted that this resulted from a hyperextension injury, typically from a fall and trauma to the head resulting in an acute spinal cord. Therefore, the presence of spinal stenosis with cervical spondylotic myelopathy in the geriatric population makes otherwise inconsequential falls more likely to be injurious to the spinal cord. Central cord injuries tend to be incomplete SCIs (American Spinal Injury Association Grades C and D), with preservation of neurological function below the lesion or area of injury. 7,10

\section{Classification and Management}

Geriatric patients with vertebral injuries and SCIs differ from younger individuals in that they are more likely to sustain incomplete neurological injuries as opposed to complete injuries. Harrop et al..$^{15}$ and DeVivo et al. ${ }^{7}$ have further demonstrated that geriatric patients are more likely to have less severe SCIs than their younger counterparts due to less severe mechanisms of injury. Three specific clinical syndromes or fracture types of the vertebrae and SCI appear to account for the majority of cases involving the geriatric population. These are as follows: 1) central cord injuries; 2) cervical extension/distraction injuries ("open book" fractures); and 3) odontoid fractures. Each injury type presents several unique management issues due to the potential for certain morbidities in the geriatric population. ${ }^{6}$

\section{Cervical CCS}

Acute traumatic CCS due to cervical spondylosis is the most common incomplete SCI, and it is considered to be a disorder primarily limited to the elderly population. Central cord injury, as originally described by Schneider et al., ${ }^{27}$ is a clinical description based on the scenario of a hyperextension trauma and weakness disproportionately greater in the arms than in the legs. In patients $>50$ years of age, the majority $(76 \%)$ of CCS cases are the result of 


\section{Geriatric spinal cord injury}
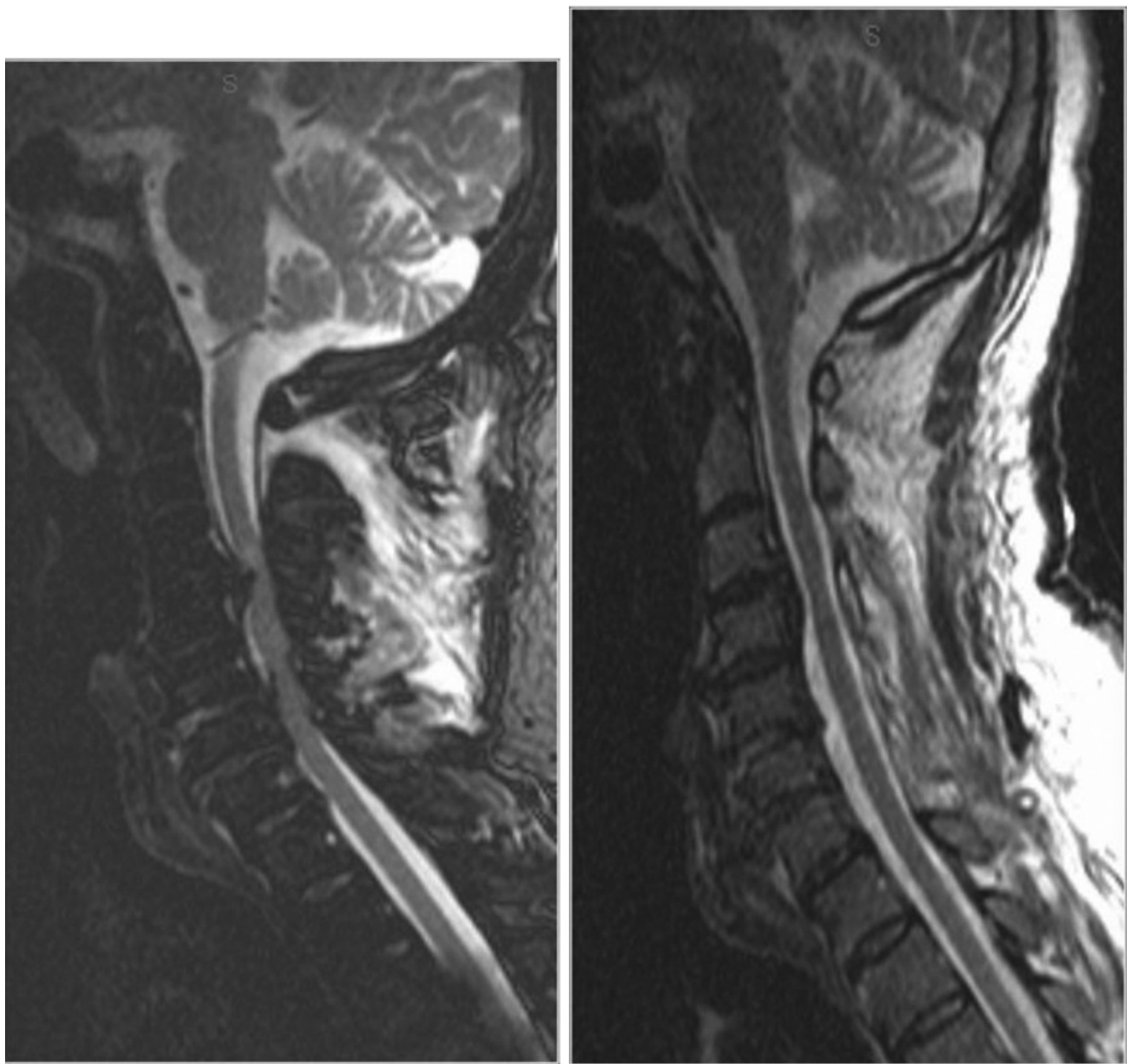

FIG. 1. Sagittal MR images demonstrating central cord injury before and after decompression in a 58-year-old man. Left: Predecompression image obtained in this patient, who suffered a fall and immediate quadriplegia that resolved to CCS in minutes. Cervical MR imaging demonstrated spondylosis and spinal cord compression. Right: An MR image obtained after posterior decompression, with the spinal cord migrating posteriorly and no further external compression seen.

hyperextension injuries in the setting of a spondylotic canal, without a bone fracture. ${ }^{30}$ Because initial radiographs typically do not illustrate fractures or acute pathological injuries, and the mechanism of injury is easily overlooked, these patients may not be accurately diagnosed and treated on initial evaluation. ${ }^{26}$ The rare patient who does display a fracture, dislocation, or evidence of cervical instability should be treated acutely with immobilization followed possibly by surgical decompression and internal fixation.

Treatment of elderly patients is aimed at weighing the risks of surgical intervention against the anticipated potential benefits as well as considering the risks and benefits of external immobilization. Although most patients with a CCS will achieve some degree of symptomatic improvement with medical or conservative therapy, surgical decompression may potentially maximize recovery and prevent future or repeated cervical SCI. There is some controversy over the optimal timing of surgical decompression in these individuals. However, it appears that prolonged spinal cord compression may result in further deterioration of neurological function and may potentially limit overall recovery.

Brodkey et al. ${ }^{4}$ illustrated that with surgical decompression there is potential for improving neurological function, even if the neurological dysfunction has been present for a prolonged period, such as several years. As described in Hagen et al., ${ }^{14}$ Guest and colleagues showed that early surgery is safe and is more cost effective than late surgery for the treatment of traumatic CCS (based on intensive care unit stay, length of stay, and improved overall motor recovery) in patients whose CCS was related to acute disc herniation or fracture. In patients with spinal stenosis or spondylosis, early surgery was safe but did not improve motor outcome compared with late surgery. Surgeons may opt to treat patients without cervical 

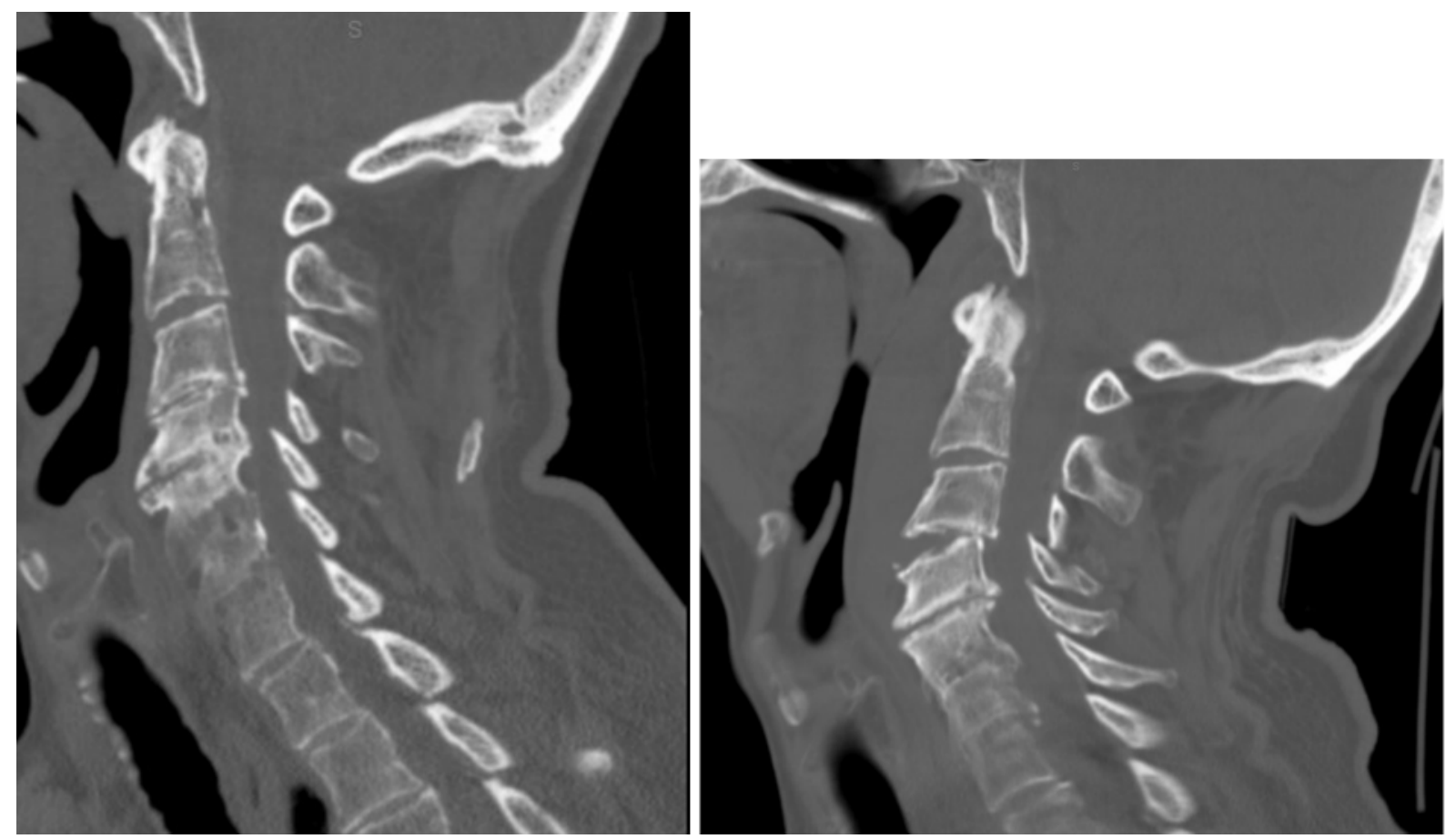

FIG. 2. Sagittal CT scans demonstrating an "open book" fracture in an 80-year-old man. Left: Preinjury study obtained before the patient fell, with no fractures apparent on this baseline cervical CT scan. Note the autofusion of the cervical spine and severe disc collapse and spondylosis. Right: Postinjury CT scan obtained 6 months later, after the patient suffered another fall. Note the distraction of the anterior column at C3-4, with retropulsion of the C-3 vertebral body into the spinal canal.

instability, fracture, or dislocation with a posterior cervical decompression procedure such as laminectomy or laminoplasty. However, a simultaneous fusion may prevent further traumatic injury to the spinal cord from dynamic injury. In addition, due to the overall spondylosis and rigidity of the elderly cervical spine, only a minimal degree of loss of ROM is noted by the patients. Regardless of the treatment scheme, patients $>45$ years of age have less recovery of neurological function than younger patients. This may reflect the effect of chronic preexisting spinal stenosis, neuronal compression, and axonal loss in elderly patients as well as the younger patients' ability to have a degree of neuron regeneration (Fig. 1).

\section{Cervical Extension/Distraction Injuries}

Cervical extension/distraction injury is another condition that tends to affect the elderly population. With increasing age, the spinal column stiffens due to disc space collapse and osteophyte formation as a consequence of degenerative spondylosis, resulting in reduced cervical ROM. In an elderly patient with a hyperextensive cervical injury or abrasions on the face or forehead, the physician should have a high index of suspicion for an extension/ distraction injury. Patients may report that their head can touch the pillow of the bed, which typically they were unable to do prior to the trauma. This fracture results in opening or lengthening of the anterior column of the spine, and has been referred to as an "open book" fracture.

Initial radiological evaluation, however, may reveal minimal changes that can be overlooked by the untrained eye. Radiographic findings on plain x-ray films and CT scans of the cervical spine may include soft tissue swelling in the prevertebral region or distraction in a spondylitic disc space. If there is a question of the presence of an injury, MR imaging typically confirms the diagnosis because it is more sensitive to soft-tissue and anterior longitudinal ligament or disc injury. Additionally, the presence of acute blood products on gradient echo MR imaging in the spinal column will further suggest disruption in the ligaments and soft-tissue structures. These fractures can be quite unstable because the anterior and posterior spinal elements may be involved. Fixation and immobilization may require surgical internal fixation of the spine. Furthermore, physicians should take extreme care of the patient with this fracture, in that they do not respond to external forces as do those with a "normal" spine. These fractures are more like fractures seen in the population with ankylosing spondylitis, in whom the fracture is the isolated motion point between two long, ankylosed lever arms. Manipulation may result in translation of the fracture and a subsequent SCI (Fig. 2).

\section{Odontoid Fractures}

A third type of spinal column injury frequently seen in elderly patients is fracture of C-2 vertebral body, and specifically the odontoid process, such as a Type II odontoid fracture. Again these fractures are the result of a fall with cranial impact. These fractures can result in the odontoid process being displaced either anteriorly or posteriorly. Because the anatomy of the spinal cord places it posterior to the $\mathrm{C}-2$ body and odontoid peg, posteriorly 


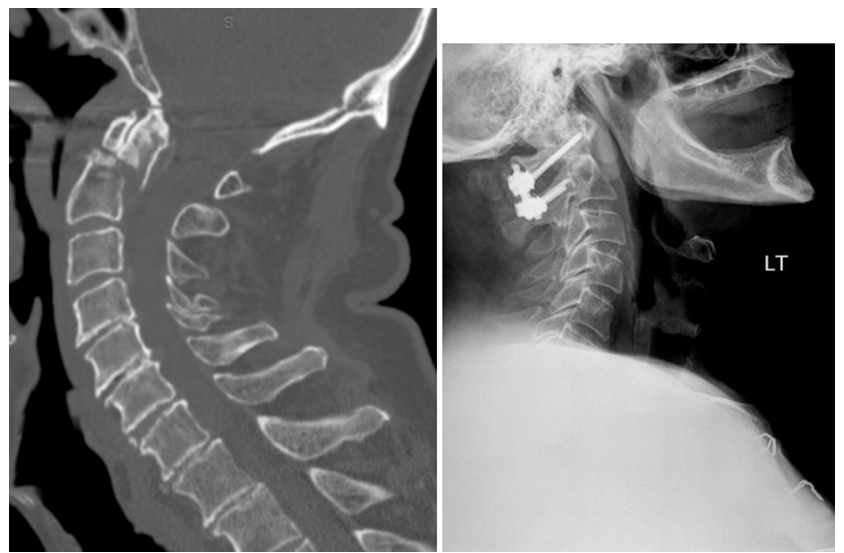

FIG. 3. Neuroimages demonstrating an odontoid fracture in a 73-year-old woman. Left: Odontoid fracture on a CT scan obtained in this patient, who had neck pain and posterior displacement of a Type II odontoid fracture. Right: Postoperative MR image demonstrating posterior C1-2 instrumented fusion in a patient with a displaced odontoid fracture after reduction of the fracture, and with normalizing of cervical alignment.

displaced odontoid fractures are more frequently associated with neurological impairment. However, despite the fact that the majority of these fractures are displaced, only a minority ( $<6 \%$ of acute odontoid fractures) cause neurological deficits, which is probably related to the large canal size at this region of the spine. ${ }^{18}$

The odontoid peg of untreated Type II odontoid fractures may migrate and can result in acute spinal cord compression, neurological deterioration, intractable pain, and possibly sudden death. Therefore, the overall treatment strategy is aimed at protecting the neural elements while restoring spinal alignment and stability and reducing pain. One technique used to reduce spinal deformity is through the application of cervical traction to reduce the fracture peg. However, in the elderly any type of flexion force may result in further airway edema and impaired respiratory mechanisms, resulting in acute airway obstruction..$^{15}$ Another option is rigid immobilization with a halo orthosis; however, as opposed to younger patients, the geriatric population often cannot tolerate this technique due to airway and swallowing dysfunction as well as the significant weight of these devices on their frames. An additional difficulty with this fracture in the elderly is the significant incidence of pseudarthrosis, even despite rigid (halo vest) immobilization techniques. Therefore, many surgeons have advocated rigid internal fixation to promote the highest rates of arthrodesis. Nevertheless, the use of a cervical collar is still an option for older patients, as long as the surgeon and patient are aware that close follow-up is needed to detect any pseudarthrosis.

A definitive treatment algorithm for geriatric patients is unclear, because both primary halo vest immobilization and surgical stabilization have significant morbidities. ${ }^{5}$ The limitations of immobilization techniques are illustrated by a significantly lower union rate of $<70 \%$, despite even halo immobilization for the geriatric population. ${ }^{21}$ In addition, halo immobilization is poorly tolerated in elderly patients, and has been reported to result in in- creased rates of the following complications: pneumonia, painful sores, decubiti, pin site cellulitis, pin loosening, swallowing dysfunction, and ultimately mobility impairment. Further studies have reported that the halo vest orthosis causes statistically significant decreases in respiratory function as measured with vital capacity, and that it may potentiate respiratory infections. ${ }^{20}$ Geriatric patients tend not to tolerate immobilization in a halo orthosis due to these adverse events.

Geriatric patients with irreducible, grossly unstable fractures or failed/closed immobilization should be considered for internal fixation. In addition, the presence of neurological deficits, polytrauma, and fractures $>6$ weeks old are relative indications for surgical treatment. Although the successful arthrodesis and immobilization rate is excellent in $88-100 \%$ of odontoid fractures, Type II odontoid fractures have the lowest rate of spontaneous fusion. ${ }^{1}$ The majority of the literature on surgical treatment of these fractures has been retrospective, and varied results have been reported, but it does appear that advanced age negatively influences fracture healing. Presently, surgical techniques with fixed cantilever instrumentation in the form of C-1 lateral mass fixation with concurrent $\mathrm{C}-2$ fixation provide solid immobilization. This technique, even in patients with osteoporosis, provides greater biomechanical fixation over earlier techniques, such as Brooke fusions, which fail in rotation and translation (Fig. 3).

\section{Outcomes}

Several trends are apparent when analyzing SCIs and their associated outcome in geriatric patients. One observation is that deaths appear to be increased in the geriatric population. ${ }^{10}$ DeVivo et al. ${ }^{8}$ reported that the 7-year survival of patients is decreased from $86.7 \%$ in the general population to $22.7 \%$ in patients with SCIs who are $>50$ years of age. In addition, patients with concomitant neuromuscular disorders, which tend to be seen more often in the elderly (that is, Parkinson disease), have also been shown to have worse outcomes. ${ }^{2}$ Furthermore, respiratory insufficiency is a common baseline deficiency in the elderly population that becomes a significant factor in the patient with SCI, resulting in an increased morbidity and mortality rate. These factors result in an overall increase in tracheostomy requirements due to decreased vital capacity, compliance, reserve volumes, and muscular ability protect the airway. ${ }^{15}$

Regarding neurological recovery and independent living status, rehabilitation produces gains in activities of daily living comparable to those seen in younger patients, ${ }^{17}$ but elderly patients unfortunately achieve less objective neurological recovery and independent walking.? Although the rehabilitation focus is more toward achieving functional independence and not necessarily toward regaining the ability to walk, immobility is not a benign process. Immobile patients are predisposed to osteoporosis, fractures, decubiti, and bone demineralization. Venous thromboembolism is yet another concern, because older patients may have additional risk factors such as a sedentary lifestyle and hypercoagulable states (that is, 


\section{P. Jabbour et al.}

malignancy), producing an overall 5-10\% increase in the risk of clinically detectable pulmonary emboli. Other potential morbidities include increased paralytic ileus; peptic ulcers, which may go unnoticed as elderly patients often will not complain of pain; and constipation, which is more common in the elderly at baseline and is further exacerbated by immobility and decreased intake of fiber and fluid during recovery. ${ }^{29}$ In addition to the fact that these conditions are more prevalent in the elderly, they are also more difficult to manage in this population. For example, loss of bladder control is more common in older patients after an SCI. However, they have more difficulty in adjusting to intermittent catheterization due to diminished hand coordination, tactile sensation, and comorbidities such as prostatic hypertrophy. ${ }^{28}$

Not all adverse consequences of SCIs are detrimental. Spasticity may have some potential benefits, including increased muscle bulk and tone, decreased osteoporosis, and the ability to ambulate and stand. ${ }^{29}$ This is balanced, however, with the adverse effects of spasticity, which include interference with transfer, wheelchair ejection, muscle pain, joint contractures, and interference with perineal care (in the case of adductor spasms). Furthermore, present treatment modalities for spasticity have significant side effects. The most used medications are baclofen and diazepam, but each can result in sedation and lethargy, which may predispose the patient to more falls and injuries. An additional agent, dantrolene, carries a significant risk of hepatitis. As with surgical treatment, these long-term medical management options must balance potential benefits against potential adverse side effects.

\section{Conclusions}

Spinal cord injuries in the elderly population are common, and have been increasing in incidence as the population ages. Consideration of the unique origins, management issues, and outcomes for injury in geriatric patients is mandated by concern for their quality of life and increasing cost to society. Because a high proportion of these injuries are incomplete SCIs and patients are often able to recover good function, striving for ideal management specific to this patient population can significantly impact patient care. However, the geriatric patient with a spinal fracture is significantly different from other adult patients with spinal fractures; thus, these populations should be stratified and considered separately in analysis of treatment pattern and outcome.

\section{Disclaimer}

The authors report no conflict of interest concerning the materials or methods used in this study or the findings specified in this paper.

\section{References}

1. Aebi M, Etter C, Coscia M: Fractures of the odontoid process. Treatment with anterior screw fixation. Spine 14:1065-1070, 1989

2. Babat LB, McLain RF, Bingaman W, Kalfas I, Young P, Rufo-
Smith C: Spinal surgery in patients with Parkinson's disease: construct failure and progressive deformity. Spine 29:20062012, 2004

3. Bracken MB, Freeman DH Jr, Hellenbrand K: Incidence of acute traumatic hospitalized spinal cord injury in the United States, 1970-1977. Am J Epidemiol 113:615-622, 1981

4. Brodkey JS, Miller CF Jr, Harmody RM: The syndrome of acute central cervical spinal cord injury revisited. Surg Neurol 14:251-257, 1980

5. Clark CR, White AA III: Fractures of the dens. A multicenter study. J Bone Joint Surg Am 67:1340-1348, 1985

6. D'Alise MD, Benzel EC: The Geriatric spine, in Benzel EC (ed): Spine surgery, 2 ed. Philadelphia: Churchill Livingstone, 2004, Vol 2, pp 1350-1355

7. DeVivo MJ, Kartus PL, Rutt RD, Stover SL, Fine PR: The influence of age at time of spinal cord injury on rehabilitation outcome. Arch Neurol 47:687-691, 1990

8. DeVivo MJ, Kartus PL, Stover SL, Rutt RD, Fine PR: Seven-year survival following spinal cord injury. Arch Neurol 44:872-875, 1987

9. Dvorak J, Froehlich D, Penning L, Baumgartner H, Panjabi MM: Functional radiographic diagnosis of the cervical spine: flexion/extension. Spine 13:748-755, 1988

10. Fassett DR, Harrop JS, Maltenfort M, Jeyamohan SB, Ratliff JD, Anderson DG, et al: Mortality rates in geriatric patients with spinal cord injuries. J Neurosurg Spine 7:277-281, 2007

11. Friedenberg ZB, Miller WT: Degenerative disc disease of the cervical spine. J Bone Joint Surg Am 45:1171-1178, 1963

12. Furlan JC, Bracken MB, Fehlings MG: Is age a key determinant of mortality and neurological outcome after acute traumatic spinal cord injury? Neurobiol Aging [epub ahead of print], 2008

13. Furlan JC, Krassioukov AV, Fehlings MG: The effects of gender on clinical and neurological outcomes after acute cervical spinal cord injury. J Neurotrauma 22:368-381, 2005

14. Hagen EM, Aarli JA, Gronning M: The clinical significance of spinal cord injuries in patients older than 60 years of age. Acta Neurol Scand 112:42-47, 2005

15. Harrop JS, Vaccaro A, Przybylski GJ: Acute respiratory compromise associated with flexed cervical traction after $\mathrm{C} 2$ fractures. Spine 26:E50-E54, 2001

16. Kannus P, Niemi S, Palvanen M, Parkkari J: Continuously increasing number and incidence of fall-induced, fracture-associated, spinal cord injuries in elderly persons. Arch Intern Med 160:2145-2149, 2000

17. Kennedy P, Evans MJ, Berry C, Mullin J: Comparative analysis of goal achievement during rehabilitation for older and younger adults with spinal cord injury. Spinal Cord 41:4452,2003

18. Kirankumar MV, Behari S, Salunke P, Banerji D, Chhabra DK, Jain VK: Surgical management of remote, isolated type II odontoid fractures with atlantoaxial dislocation causing cervical compressive myelopathy. Neurosurgery 56:10041012, 2005

19. Krassioukov AV, Furlan JC, Fehlings MG: Medical co-morbidities, secondary complications, and mortality in elderly with acute spinal cord injury. J Neurotrauma 20:391-399, 2003

20. Lind B, Bake B, Lundqvist C, Nordwall A: Influence of halo vest treatment on vital capacity. Spine 12:449-452, 1987

21. Maiman DJ, Larson SJ: Management of odontoid fractures. Neurosurgery 11:471-476, 1982

22. Miller KE, Zylstra RG, Standridge JB: The geriatric patient: a systematic approach to maintaining health. Am Fam Physician 61:1089-1104, 2000

23. O'Connor PJ: Trends in spinal cord injury. Accid Anal Prev 38:71-77, 2006

24. Richardson JK, Hurvitz EA: Peripheral neuropathy: a true 


\section{Geriatric spinal cord injury}

risk factor for falls. J Gerontol A Biol Sci Med Sci 50: M211-M215, 1995

25. Sattin RW: Falls among older persons: a public health perspective. Annu Rev Public Health 13:489-508, 1992

26. Scher AT: Hyperextension trauma in the elderly: an easily overlooked spinal injury. J Trauma 23:1066-1068, 1983

27. Schneider RC, Cherry G, Pantek H: The syndrome of acute central cervical spinal cord injury; with special reference to the mechanisms involved in hyperextension injuries of cervical spine. J Neurosurg 11:546-577, 1954

28. Scivoletto G, Morganti B, Ditunno P, Ditunno JF, Molinari M: Effects on age on spinal cord lesion patients' rehabilitation. Spinal Cord 41:457-464, 2003

29. Teasell R, Allatt D: Managing the growing number of spinal cord-injured elderly. Geriatrics 46:78, 83-85, 89, 1991

30. Weingarden SI, Graham PM: Falls resulting in spinal cord injury: patterns and outcomes in an older population. Paraplegia 27:423-427, 1989
31. Teresi LM, Lufkin RB, Reicher MA, Moffit BJ, Vinuela FV, Wilson GM, et al: Asymptomatic degenerative disk disease and spondylosis of the cervical spine: MR imaging. Radiology 164:83-88, 1987

32. Young WF: Cervical spondylotic myelopathy: a common cause of spinal cord dysfunction in older persons. Am Fam Physician 62:1064-1070, 1073, 2000

Manuscript submitted July 14, 2008

Accepted November 5, 2008.

Address correspondence to: James S. Harrop, M.D., Department of Neurological Surgery, Thomas Jefferson University, 909 Walnut Street, Philadelphia, Pennsylvania 19107. email: James.harrop@ jefferson.edu. 